\title{
HYDRODYNAMIC FLOW BETWEEN ROTATING ECCENTRIC CYLINDERS WITH SUCTION AT THE POROUS WALLS
}

\author{
S. MEENA, P. KANDASWAMY, and LOKENATH DEBNATH
}

(Received 19 November 1998)

\begin{abstract}
The flow of a viscous, incompressible fluid between two eccentric rotating porous cylinders with suction/injection at both the cylinders, for very small clearance ratio is studied. The expressions for various flow characteristics are obtained using perturbation analysis. Streamlines and pressure plots are shown graphically for various values of flow parameters and discussed.
\end{abstract}

2000 Mathematics Subject Classification. Primary 76D08.

1. Introduction. In the present investigation we have considered the viscous flow between eccentric porous rotating cylinders. The effect of uniform suction and injection is studied on the walls of the cylinders. Several authors have analysed flow through rotating eccentric cylinders for various flow situations. In particular the complex hydrodynamical lubrication problem as flow between two rotating eccentric cylinders was analysed by Reynolds [10]. Wannier [13] presented an exact solution of the Stokes equation for the flow between rotating eccentric cylinders. Here, the pressure distribution reduces to the classical Sommerfeld solution of the Reynolds equation in the limit of small clearance ratio, and provides a correction for curvature effects. Wood [14] has studied the asymptotic expansion at large Reynolds number for steady motion between non-coaxial eccentric rotating cylinders using modified bipolar coordinate system. Kamal [6] has considered the effect of the inertial terms on the flow between rotating eccentric cylinders. As no restriction was laid on the clearance ratio, the analysis is somewhat complicated when applied to the lubrication problem. Kulinski and Ostrach [7] also have considered the effect of inertia on the flow in a journal bearing by using a perturbation procedure for small eccentricity. Sood and Elrod [12] have used numerical techniques to solve the full Navier-Stokes equations for the flow between eccentric rotating cylinders but for a clearance ratio of 1.0 only. DiPrima and Stuart [3] have studied the hydrodynamic flow between rotating eccentric cylinders using the modified bipolar coordinate system. They derived the Sommerfeld pressure distribution and associated flow from the Navier-Stokes equation by a straightforward and systematic expansion in the clearance ratio (assumed small).

Dintenfass [2] considered the role of cartilage elasticity coupled with the nonnewtonian behaviour of synovial fluid to explain the joint lubrication. Dawson [4] suggested the existence of more than one mode of lubrication namely boundary lubrication, and this idea was supported by McCutchen [9] who concluded that weeping lubrication was not the sum total of joint lubrication but was supplemented with 
boundary lubrication. Fein [5] was first to give a mathematical model for synovial joints. He concludes that the joint is hydrodynamically lubricated with other phenomena such as the non-newtonian nature of the fluid weeping, playing a secondary role. Ling [8] presented an analytical model to account for non-linearity of cartilage. He considered the fluid filled cartilage as a composite which consists of (i) a rigid substrate, and (ii) a fluid filled matrix layer. The two surfaces were modelled as two circular porous discs which were pressed with the fluid film in between. The movement of the synovial fluid in porous disc was taken to be governed by Darcy's law. Chandra [1] considered the porous plate model for synovial joint under squeezing. Sambasiva Rao et al. [11] have studied the effect of thin film of synovial fluid contained between slider bearings. Hence the problem of lubricated contact of the cartilage surface is very complex and so only a step-by-step study seems to be feasible in this direction.

One of the most important area for the application of the problem is the human (synovial) joints. Synovial joints provided by nature in human body to carry out the trouble free motion of a bone past another have long been identified as bearing system. In fact these joints function as excellent bearings in tribological conditions. From an engineering point of view, the synovial joints are weight bearing systems operating under a wide range of loading conditions. The mechanism of the synovial joint is akin to any bearing system in tribological conditions. Finally, even the simplest model of the synovial joint must take into consideration the porosity of the cartilage and the eccentricity of the two surfaces involved. Hence, here an attempt on the flow between two eccentric rotating porous cylinders is made. To be exact in finding the effects of the porosity on the flow field on a system, consider the flow in the porous media and the slip in the tangential velocity field that one will encounter at the porous interface. But here as the thickness of the porous medium in the human bones is very thin, it is assumed that there is no tangential flow of lubricating fluid in the porous medium and hence the slip expected at the porous interface is absent, thus considering only a normal flow of the lubricating fluid into the porous cartilage. It is assumed that the normal velocity of the fluid in the porous cartilage is uniform. These normal velocities at the inner and outer cylinders are taken as $v$ and $u$, respectively. Expressions for various flow characteristics are obtained using perturbation analysis.

2. Mathematical analysis. We consider a viscous, incompressible fluid confined between two infinitely long circular cylinders of radii $a$ and $b(b>a)$, with centers set a distance " $a e$ " apart. In order to ensure that the two cylinders do not touch each other we have,

$$
0 \leq \epsilon \leq 1
$$

where

$$
\epsilon=\frac{e}{\delta}, \quad \delta=\frac{b-a}{a} .
$$

The parameter $\epsilon$ is the eccentricity and $\delta$ the clearance ratio. The polar coordinate 


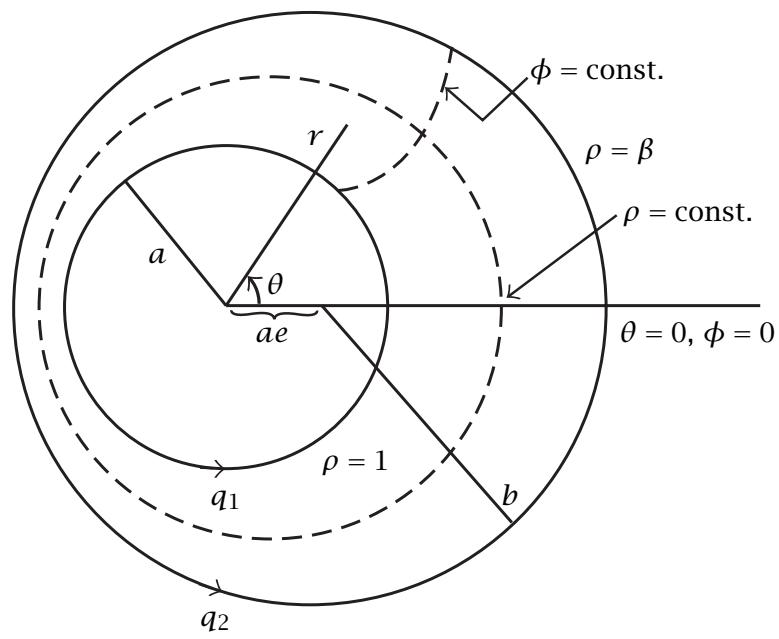

FIGURE 2.1. Geometry and coordinate systems.

system shown in Figure 2.1 has its origin at the axis of the inner cylinder with the ray $\theta=0$ through the axis of the outer cylinder. As in [14], the modified bipolar coordinates are introduced by means of the conformal transformation

$$
Z=\frac{(a \zeta+\gamma)}{1+\gamma \zeta}, \quad Z=r e^{i \theta}, \zeta=\rho e^{i \phi},
$$

where

$$
\begin{gathered}
\gamma=\frac{-(1+\beta)+\left[(1+\beta)^{2}-4 \epsilon^{2} \beta\right]^{1 / 2}}{2 \epsilon \beta}, \\
\beta=\frac{[1+\delta+\epsilon \delta-\gamma]}{1-(1+\delta) \gamma-\epsilon \gamma \delta} .
\end{gathered}
$$

The coordinate curves $\rho$ equal to constant are circles; in particular the inner and outer cylinders are given by $\rho=1$ and $\rho=\beta$, respectively. An advantage of the modified bipolar coordinate system as compared to the usual bipolar coordinate system is that, in the limit $\epsilon \rightarrow 0$, the $\rho, \phi$ coordinate system reduces to the $r, \theta$ coordinate system except for a scale factor of $a$. The Jacobian $J$ of the transformation (2.3) is given by

$$
J=\frac{\left(1+2 \gamma \rho \cos \phi+\gamma^{2} \rho^{2}\right)^{2}}{\left(1-\gamma^{2}\right)^{2}} .
$$

The element of arc length in two dimensions is

$$
d s^{2}=d r^{2}+r^{2} d \theta^{2}=\frac{a^{2}}{J} d \rho^{2}+\frac{a^{2} \rho^{2}}{J} d \phi^{2} .
$$

Let $u_{\rho}$ and $u_{\phi}$ denote the velocity components in the $\rho$ and $\phi$ directions, respectively, and let $p$ be the pressure, $\sigma$ the density, and $v$ the kinematic viscosity. Then the 
momentum and continuity equations are

$$
\begin{aligned}
& \frac{\partial u_{\rho}}{\partial t}+ \frac{\sqrt{J}}{a} u_{\rho} \frac{\partial u_{\rho}}{\partial \rho}+\frac{\sqrt{J}}{a \rho} u_{\phi} \frac{\partial u_{\rho}}{\partial \phi}-\frac{1}{a} \frac{\partial}{\partial \phi}\left(\frac{\sqrt{J}}{\rho}\right) u_{\rho} u_{\phi}+\frac{\rho}{a} \frac{\partial}{\partial \rho}\left(\frac{\sqrt{J}}{\rho}\right) u_{\phi}^{2} \\
&=-\frac{\sqrt{J}}{a \sigma} \frac{\partial p}{\partial \rho}+\frac{v \sqrt{J}}{a^{2}} \frac{\partial}{\partial \rho}\left[\frac{J}{\rho} \frac{\partial}{\partial \rho}\left(\frac{\rho u_{\rho}}{\sqrt{J}}\right)\right]+\frac{1}{\rho^{2}} \frac{\partial}{\partial \phi}\left[J \frac{\partial}{\partial \phi}\left(\frac{u_{\rho}}{\sqrt{J}}\right)\right] \\
&+\frac{v}{a^{2}}\left(-\frac{2 J}{\rho^{2}} \frac{\partial u_{\phi}}{\partial \phi}+\frac{1}{\rho} \frac{\partial J}{\partial \rho} \frac{\partial u_{\phi}}{\partial \phi}-\frac{1}{\rho} \frac{\partial J}{\partial \phi} \frac{\partial u_{\phi}}{\partial \rho}\right), \\
& \frac{\partial u_{\phi}}{\partial t}+ \frac{\sqrt{J}}{a} u_{\rho} \frac{\partial u_{\phi}}{\partial \rho}+\frac{\sqrt{J}}{a \rho} u_{\phi} \frac{\partial u_{\phi}}{\partial \phi}-\frac{\rho}{a} \frac{\partial}{\partial \phi}\left(\frac{\sqrt{J}}{\rho}\right) u_{\rho} u_{\phi}+\frac{1}{a} \frac{\partial}{\partial \phi}\left(\frac{\sqrt{J}}{\rho}\right) u_{\rho}^{2} \\
&=-\frac{\sqrt{J}}{a \sigma \rho} \frac{\partial p}{\partial \phi}+\frac{v \sqrt{J}}{a^{2}} \frac{\partial}{\partial \rho}\left[\frac{J}{\rho} \frac{\partial}{\partial \rho}\left(\frac{\rho u_{\phi}}{\sqrt{J}}\right)\right]+\frac{1}{\rho^{2}} \frac{\partial}{\partial \phi}\left[J \frac{\partial}{\partial \phi}\left(\frac{u_{\phi}}{\sqrt{J}}\right)\right] \\
&+\frac{v}{a^{2}}\left(\frac{2 J}{\rho^{2}} \frac{\partial u_{\rho}}{\partial \phi}-\frac{1}{\rho} \frac{\partial J}{\partial \rho} \frac{\partial u_{\rho}}{\partial \phi}+\frac{1}{\rho} \frac{\partial J}{\partial \phi} \frac{\partial u_{\rho}}{\partial \rho}\right), \\
& \frac{\partial}{\partial \rho}\left(\frac{\rho}{\sqrt{J}} u_{\rho}\right)+\frac{\partial}{\partial \phi}\left(\frac{u_{\phi}}{\sqrt{J}}\right)=0 .
\end{aligned}
$$

The boundary conditions are

$$
\begin{aligned}
& u_{\phi}=q_{1}, \quad u_{\rho}=v, \quad \text { at } \rho=1 \text {, } \\
& u_{\phi}=q_{2}, \quad u_{\rho}=u, \quad \text { at } \rho=\beta \text {. }
\end{aligned}
$$

From the continuity (2.10) a stream function $\psi$ can be introduced with

$$
u_{\rho}=\frac{\sqrt{J}}{\rho} \frac{\partial \psi}{\partial \phi}, \quad u_{\phi}=-\sqrt{J} \frac{\partial \psi}{\partial \rho} .
$$

Assuming the flow to be steady and by using the vector form of the vorticity equation, the vorticity $\omega$ in the axial direction is given by

$$
\frac{1}{\rho}\left(\frac{\partial \psi}{\partial \phi} \frac{\partial \Omega}{\partial \rho}-\frac{\partial \psi}{\partial \rho} \frac{\partial \Omega}{\partial \phi}\right)=\frac{v}{a}\left(\frac{\partial^{2}}{\partial \rho^{2}}+\frac{1}{\rho} \frac{\partial}{\partial \rho}+\frac{1}{\rho^{2}} \frac{\partial^{2}}{\partial \phi^{2}}\right) \omega
$$

where

$$
\omega=-\frac{J}{a}\left(\frac{\partial^{2}}{\partial \rho^{2}}+\frac{1}{\rho} \frac{\partial}{\partial \rho}+\frac{1}{\rho^{2}} \frac{\partial^{2}}{\phi^{2}}\right) \psi .
$$

Using the fact that, in lubrication problems the clearance ratio $\delta$ is small and also to facilitate the necessary algebraic calculations, it is convenient to choose $\alpha=\beta-1$ as the length scale. Equations (2.4) and (2.5) reveal

$$
\alpha(\delta, \epsilon)=\beta-1=\delta \sqrt{1-\epsilon^{2}}\left[1-\frac{1}{2} \delta\left(1-\sqrt{1-\epsilon^{2}}\right)\right]+O\left(\delta^{3}\right),
$$

for $\delta$ small. Hence $\alpha$ is uniformly small for all $\epsilon, 0 \leq \epsilon \leq 1$, when $\delta$ is small.

If we let

$$
\rho=1+\alpha x
$$

and introduce the following non-dimensional quantities

$$
\psi=\alpha q_{1} \Psi, \quad \omega=\frac{q_{1} \Omega}{a \alpha}, \quad u=\alpha q_{2} U, \quad v=\alpha q_{2} V
$$


Then

$$
u_{\rho}=\frac{\alpha q_{1} \sqrt{J}}{\rho} \frac{\partial \psi}{\partial \phi}, \quad u_{\phi}=-q_{1} \sqrt{J} \frac{\partial \psi}{\partial x} .
$$

Now equations (2.13) and (2.14) take the form

$$
(1+\alpha x)^{-1} R_{M}\left(\frac{\partial \Psi}{\partial \phi} \frac{\partial \Omega}{\partial x}-\frac{\partial \Psi}{\partial x} \frac{\partial \Omega}{\partial \phi}\right)=\left(\frac{\partial^{2}}{\partial x^{2}}+\frac{\alpha}{1+\alpha x} \frac{\partial}{\partial x}+\frac{\alpha^{2}}{(1+\alpha x)^{2}} \frac{\partial^{2}}{\partial \phi^{2}}\right) \Omega,
$$

with

$$
\Omega=-J\left(\frac{\partial^{2}}{\partial x^{2}}+\frac{\alpha}{1+\alpha x} \frac{\partial}{\partial x}+\frac{\alpha^{2}}{(1+\alpha x)^{2}} \frac{\partial^{2}}{\partial \phi^{2}}\right) \Psi .
$$

The parameter $R_{M}$ is the modified Reynolds number given by

$$
R_{M}=\frac{q_{1} a}{v} \alpha^{2} .
$$

Consistent with the scaling (2.16), the pressure $p$ takes the form

$$
p=\frac{\mu q_{1}}{a \alpha^{2}} P,
$$

where $\mu=\sigma v$ is the viscosity and $P$ is the dimensionless pressure.

The boundary conditions (2.11) become

$$
\begin{array}{lll}
\frac{\partial \Psi}{\partial x}=-\frac{1}{\sqrt{J}}, & \frac{\partial \Psi}{\partial \phi}=\frac{\eta \rho}{\sqrt{J}} V & \text { at } x=0, \\
\frac{\partial \Psi}{\partial x}=-\frac{\eta}{\sqrt{J}}, & \frac{\partial \Psi}{\partial \phi}=\frac{\eta \rho}{\sqrt{J}} U & \text { at } x=1,
\end{array}
$$

where $\eta=q_{2} / q_{1}$.

From the above it is clear that the functional form of $\Psi$ and $P$ is

$$
\Psi=\Psi\left(x, \phi ; \epsilon, \eta, \alpha, R_{M}\right), \quad P=P\left(x, \phi ; \epsilon, \eta, \alpha, R_{M}\right) .
$$

Expanding $J$ in powers of $\alpha$, we find that

$$
J(x, \phi ; \epsilon, \alpha)=J_{0}(\phi ; \epsilon)+\alpha J_{1}(x, \phi ; \epsilon)+o\left(\alpha^{2}\right),
$$

where

$$
\begin{aligned}
J_{0}(\phi ; \epsilon) & =\frac{(1-\epsilon \cos \phi)^{2}}{1-\epsilon^{2}}, \\
J_{1}(x, \phi ; \epsilon) & =\frac{2(1-\epsilon \cos \phi)}{\sqrt{1-\epsilon^{2}}}\left[-\frac{\epsilon(\epsilon-\cos \phi)}{2\left(1-\epsilon^{2}\right)}+\frac{1-\epsilon \cos \phi-\sqrt{1-\epsilon^{2}}}{\sqrt{1-\epsilon^{2}}} x\right] .
\end{aligned}
$$

The expansions for $\Psi$ and $P$ are of the form

$$
\begin{aligned}
& \Psi\left(x, \phi ; \epsilon, \eta, \alpha, R_{M}\right) \\
& \quad=\Psi_{00}(x, \phi ; \epsilon, \eta)+R_{M} \Psi_{10}(x, \phi ; \epsilon, \eta)+\alpha \Psi_{01}(x, \phi ; \epsilon, \eta)+O\left(\alpha^{2}, R_{M}^{2}, \alpha R_{M}\right) \\
& P\left(x, \phi ; \epsilon, \eta, \alpha, R_{M}\right) \\
& \quad=P_{00}(x, \phi ; \epsilon, \eta)+R_{M} P_{10}(x, \phi ; \epsilon, \eta)+\alpha P_{01}(x, \phi ; \epsilon, \eta)+O\left(\alpha^{2}, R_{M}^{2}, \alpha R_{M}\right) .
\end{aligned}
$$


The expansion of $\Omega$, obtained from equation (2.20), is

$$
\begin{aligned}
& \Omega\left(x, \phi ; \epsilon, \eta, \alpha, R_{M}\right) \\
& \quad=\Omega_{00}(x, \phi ; \epsilon, \eta)+R_{M} \Omega_{10}(x, \phi ; \epsilon, \eta)+\alpha \Omega_{01}(x, \phi ; \epsilon, \eta)+O\left(\alpha^{2}, R_{M}^{2}, \alpha R_{M}\right) \\
& \quad=-J_{0} \frac{\partial^{2} \Psi_{00}}{\partial x^{2}}-R_{M} J_{0} \frac{\partial^{2} \Psi_{10}}{\partial x^{2}}-\alpha\left[J_{0} \frac{\partial^{2} \Psi_{01}}{\partial x^{2}}+J_{1} \frac{\partial^{2} \Psi_{00}}{\partial x^{2}}+J_{0} \frac{\partial \Psi_{00}}{\partial x}\right] .
\end{aligned}
$$

Substituting these expansions into the differential equation (2.19) and the boundary conditions (2.23) and making use of the expansion of $J$, we obtain three problems for $\Psi_{00}, \Psi_{10}, \Psi_{01}$ given in the following. In addition, it is necessary to make use of the condition that the pressure $P$ is singlevalued in $\phi$. The appropriate equations for $P_{00}$, $P_{10}, P_{01}$, respectively, are obtained by introducing dimensionless variables in (2.9) and then substituting the expansions (2.28) and (2.29) for $\Psi$ and $P$.

THE REYNOLDS APPROXIMATION: $\alpha=0, R_{M}=0$.

$$
\begin{aligned}
\frac{\partial^{4} \Psi_{00}}{\partial x^{4}} & =0, \\
\frac{\partial \Psi_{00}}{\partial \phi} & =\frac{\eta V \sqrt{1-\epsilon^{2}}}{1-\epsilon \cos \phi}, \quad \frac{\partial \Psi_{00}}{\partial x}=\frac{\sqrt{1-\epsilon^{2}}}{1-\epsilon \cos \phi} \quad \text { at } x=0, \\
\frac{\partial \Psi_{00}}{\partial \phi} & =\frac{\eta U \sqrt{1-\epsilon^{2}}}{1-\epsilon \cos \phi}, \quad \frac{\partial \Psi_{00}}{\partial x}=-\frac{\eta \sqrt{1-\epsilon^{2}}}{1-\epsilon \cos \phi} \quad \text { at } x=1, \\
\frac{\partial P_{00}}{\partial \phi} & =-J_{0} \frac{\partial^{3} \Psi_{00}}{\partial x^{3}} .
\end{aligned}
$$

THE INERTIAL OR $R_{M}$ CORRECTION: $\alpha=0, R_{M} \neq 0$.

$$
\begin{aligned}
& \frac{\partial^{4} \Psi_{10}}{\partial x^{4}}=\frac{\partial^{3} \Psi_{00}}{\partial x^{3}} \frac{\partial \Psi_{00}}{\partial \phi}-\frac{1}{J_{0}} \frac{\partial \Psi_{00}}{\partial x} \frac{\partial}{\partial \phi}\left(J_{0} \frac{\partial^{2} \Psi_{00}}{\partial x^{2}}\right), \\
& \frac{\partial \Psi_{10}}{\partial x}=\frac{\partial \Psi_{10}}{\partial \phi}=0 \quad \text { at } x=0, x=1, \\
& \frac{\partial P_{10}}{\partial \phi}=-J_{0} \frac{\partial^{3} \Psi_{10}}{\partial x^{3}}+\sqrt{J_{0}}\left(\frac{\partial \Psi_{00}}{\partial \phi} \frac{\partial}{\partial x}-\frac{\partial \Psi_{00}}{\partial x} \frac{\partial}{\partial \phi}\right)\left(\sqrt{J_{0}} \frac{\partial \Psi_{00}}{\partial x}\right) .
\end{aligned}
$$

THE CURVATURE OR $\alpha$ CORRECTION: $\alpha \neq 0, R_{M}=0$.

$$
\frac{\partial^{4} \Psi_{01}}{\partial x^{4}}=-2 \frac{\partial^{3} \Psi_{00}}{\partial x^{3}}-\frac{1}{J_{0}} \frac{\partial^{2}}{\partial x^{2}}\left(J_{1} \frac{\partial^{2} \Psi_{00}}{\partial x^{2}}\right),
$$




$$
\begin{aligned}
\frac{\partial \Psi_{01}}{\partial \phi} & =\frac{\eta V \epsilon(\epsilon-\cos \phi)}{2(1-\epsilon \cos \phi)^{2}}, \\
\frac{\partial \Psi_{01}}{\partial x} & =-\frac{\epsilon(\epsilon-\cos \phi)}{2(1-\epsilon \cos \phi)^{2}}=M(\phi) \quad \text { at } x=0, \\
\frac{\partial \Psi_{01}}{\partial \phi} & =\frac{\eta U\left(2-\epsilon^{2}-\epsilon \cos \phi\right)}{2(1-\epsilon \cos \phi)^{2}} \quad \text { at } x=1, \\
\frac{\partial \Psi_{01}}{\partial x} & =-\eta\left[\frac{\left(2-\epsilon^{2}-\epsilon \cos \phi\right)}{2(1-\epsilon \cos \phi)^{2}}-\frac{\sqrt{1-\epsilon^{2}}}{(1-\epsilon \cos \phi)}\right]=N(\phi) \quad \text { at } x=1, \\
\frac{\partial P_{01}}{\partial \phi} & =\left(x+\frac{J_{1}}{2 J_{0}}\right) \frac{\partial P_{00}}{\partial \phi}-J_{0} \frac{\partial^{2}}{\partial x^{2}}\left(\frac{\partial \Psi_{01}}{\partial x}+\frac{J_{1}}{2 J_{0}} \frac{\partial \Psi_{00}}{\partial x}=\Psi_{00}\right) .
\end{aligned}
$$

3. Solutions of the successive problems. In this section, the solution of the above three problems are given.

THE REYNOLDS APPROXIMATION: $\alpha=0, R_{M}=0$. The general solution of (2.31) is

$$
\Psi_{00}(x, \phi)=\sum_{n=0}^{3} A_{00 n}(\phi) x^{n} .
$$

The functions $A_{00 n}$ are determined so that the boundary conditions (2.32) are satisfied and so that $P_{00}$ is periodic and singlevalued. This latter condition requires that $\int_{0}^{2 \pi}\left(\partial P_{00} / \partial \phi\right) d \phi=0$.

We find

$$
\begin{aligned}
\Psi_{00}(x, \phi)= & -\frac{\sqrt{1-\epsilon^{2}}}{2+\epsilon^{2}}(\eta+1)\left(3 x^{2}-2 x^{3}\right) \\
& +\frac{\sqrt{1-\epsilon^{2}}}{1-\epsilon \cos \phi}\left[-x+(\eta+2) x^{2}-(\eta+1) x^{3}\right] \\
& +2 \eta k(\phi)\left[V+3(U-V) x^{2}-2(U-V) x^{3}\right], \\
P_{00}(x, \phi)= & \frac{24 \eta(U-V)}{\left(1-\epsilon^{2}\right)}\left[-2 \epsilon \sin \phi+\frac{\epsilon^{2}}{4} \sin 2 \phi+\frac{1}{2}\left(2+\epsilon^{2}\right) \phi\right] K(\phi) \\
& +\frac{2+\epsilon^{2}}{2} L(\phi)+\frac{3}{4} \sqrt{1-\epsilon^{2}} \log (1-\epsilon \cos \phi)+\frac{\sqrt{1-\epsilon^{2}}}{4}(1-\epsilon \cos \phi) \\
& +\frac{6 \epsilon(\eta+1)\left(2-\epsilon^{2}-\epsilon \cos \phi\right) \sin \phi}{\left(2+\epsilon^{2}\right) \sqrt{1-\epsilon^{2}}}+G_{00},
\end{aligned}
$$

where

$$
K(\phi)=\tan ^{-1} \sqrt{\frac{1+\epsilon}{1-\epsilon}} \tan \left(\frac{\phi}{2}\right), \quad L(\phi)=\int K(\phi) d \phi,
$$

$A_{000}$ and $G_{00}$ are arbitrary constants. Note that $\Psi_{00}$ is symmetric and the pressure $P_{00}$ is antisymmetric where the line $\phi=0$.

To show that (3.3) is truly the Sommerfeld pressure distribution it is necessary to convert from the $\phi$ coordinate of the modified bipolar coordinate system to the $\theta$ coordinate of the polar coordinate system. This is done as follows. On the circle $\rho=1$, $r=a$, it follows from (2.5) that

$$
d \theta=\left.\frac{1}{\sqrt{J}}\right|_{\rho=1} d \phi .
$$


Substituting for $J$ from equations (2.24), (2.25), and (2.26) we obtain

$$
\int_{0}^{\theta} d \theta=\int_{0}^{\phi}\left[\frac{\sqrt{1-\epsilon^{2}}}{1-\epsilon \cos \phi}+\alpha \frac{\epsilon(\epsilon-\cos \phi)}{2(1-\epsilon \cos \phi)^{2}}+O\left(\alpha^{2}\right)\right] d \phi
$$

The integral on the right-hand side of (3.6) can be evaluated by using Sommerfeld transformation, $1+\epsilon \cos X=\left(1-\epsilon^{2}\right) /(1-\epsilon \cos \phi)$. We find that,

$$
\theta=X-\alpha \frac{2 \sin \chi}{e \sqrt{1-\epsilon^{2}}}+O\left(\alpha^{2}\right)
$$

In the limit $\alpha \rightarrow 0$, we have $\theta=\chi$ and $1+\epsilon \cos \theta=\left(1-\epsilon^{2}\right) /(1-\epsilon \cos \phi)$. It follows that

$$
\cos \phi=\frac{\epsilon+\cos \theta}{1+\cos \theta}, \quad \sin \phi=\frac{\sqrt{1-\epsilon^{2}} \sin \theta}{1+\epsilon \cos \theta}
$$

Using (3.8) and (2.15) to express $\alpha$ in terms of $\delta$, we have from (3.3) and (2.22),

$$
\begin{aligned}
& p-p_{0}=\frac{\mu q_{1}}{a \delta^{2}\left(1-\epsilon^{2}\right)} P_{000} \\
& =\frac{6 \epsilon \mu q_{1} a(\eta+1)(2+\epsilon \cos \theta) \sin \theta}{(b-a)^{2}\left(2+\epsilon^{2}\right)^{2}(1+\epsilon \cos \theta)^{2}}+\frac{\mu q_{1} a}{(b-a)^{2}\left(1-\epsilon^{2}\right)} \\
& \times \frac{24 \eta(U-V)}{\left(1-\epsilon^{2}\right)}\left\{\left[-2 \epsilon \sin \phi+\frac{\epsilon^{2}}{4} \sin 2 \phi+\frac{1}{2}\left(2+\epsilon^{2}\right) \phi\right] K(\phi)\right. \\
& +\frac{2+\epsilon^{2}}{2} L(\phi)+\frac{3}{4} \sqrt{1-\epsilon^{2}} \log (1-\epsilon \cos \phi) \\
& \left.+\frac{\sqrt{1-\epsilon^{2}}}{4}(1-\epsilon \cos \phi)\right\}+O\left(\alpha, R_{M}\right),
\end{aligned}
$$

where $p_{0}$ is a constant. When the outer cylinder is at rest, that is, $\eta=0$ this is the Sommerfeld pressure distribution.

THE INERTIAL OR $R_{M}$ CORRECTION: $\alpha=0, R_{M} \neq 0$. Having obtained $\Psi_{00}$, the righthand side of (2.34) is given as follows:

$$
\begin{aligned}
& -\frac{2 \epsilon\left(1-\epsilon^{2}\right) \sin \phi}{(1-\epsilon \cos \phi)^{2}} \sum_{n=0}^{3} B_{10 n}(\phi) x^{n}+\left[V+3(U-V) x^{2}-2(U-V) x^{3}\right] C_{100}(\phi) \\
& +\left[x-(\eta+2) x^{2}+(\eta+1) x^{3}\right] D_{100}(\phi)-\left(x^{2}-x\right) E_{100}(\phi)-\left(x^{3}-x^{2}\right) F_{100}(\phi) \\
& +\left[6(\eta+1) x^{3}-(7 \eta+11) x^{2}+2(\eta+3) x-1\right] H_{100}(\phi)-\left(12 x^{3}-18 x^{2}+6 x\right) M_{100}(\phi),
\end{aligned}
$$


where

$$
\begin{aligned}
& B_{100}(\phi)=\frac{6(\eta+1)}{2+\epsilon^{2}}-\frac{\eta+2}{1-\epsilon \cos \phi}, \\
& B_{101}(\phi)=-\frac{18(\eta+1)(\eta+3)}{2+\epsilon^{2}}-\frac{36(\eta+1)^{2}(1-\epsilon \cos \phi)}{\left(2+\epsilon^{2}\right)^{2}}+\frac{2\left(7+7 \eta+\eta^{2}\right)}{1-\epsilon \cos \phi} \\
& B_{102}(\phi)=\frac{36(\eta+1)(3+2 \eta)}{2+\epsilon^{2}}-\frac{108(\eta+1)^{2}(1-\epsilon \cos \phi)}{\left(2+\epsilon^{2}\right)^{2}}-\frac{12(\eta+1)(\eta+2)}{1-\epsilon \cos \phi}, \\
& B_{103}(\phi)=12(\eta+1)^{2}\left[-\frac{5}{2+\epsilon^{2}}+\frac{1}{1-\epsilon \cos \phi}+\frac{6(1-\epsilon \cos \phi)}{\left(2+\epsilon^{2}\right)^{2}}\right] \\
& \left.C_{100}(\phi)=\frac{\eta \sqrt{1-\epsilon^{2}}}{1-\epsilon \cos \phi}-24 \eta(U-V) K(\phi)-\frac{6 \sqrt{1-\epsilon^{2}}(\eta+1)}{1-\epsilon \cos \phi}+\frac{12(\eta+1) \sqrt{1-\epsilon^{2}}}{\left(2+\epsilon^{2}\right)}\right] \\
& D_{100}(\phi)=-\frac{24 \eta(U-V) K(\phi) \epsilon \sqrt{1-\epsilon^{2}} \sin (\phi)}{(1-\epsilon \cos \phi)^{2}}, \\
& E_{100}(\phi)=-\frac{12 \eta\left(1-\epsilon^{2}\right)(U-V) K(\phi)}{(1-\epsilon \cos \phi)^{2}}\left\{\begin{array}{c}
\frac{24 \eta(U-V)(1-\epsilon \cos \phi) \epsilon \sin \phi}{1-\epsilon^{2}} K(\phi) \\
+\frac{2 \epsilon(\eta+2) \sin \phi}{\sqrt{1-\epsilon^{2}}+\frac{6 \eta(U-V)(1-\epsilon \cos \phi)}{\sqrt{1-\epsilon^{2}}}}
\end{array}\right.
\end{aligned}
$$

$$
\begin{aligned}
F_{100}(\phi)=-\frac{12 \eta \sqrt{1-\epsilon^{2}}(U-V) K(\phi)}{(1-\epsilon \cos \phi)^{2}}\{ & -\frac{48 \epsilon \eta(U-V)(1-\epsilon \cos \phi) \sin \phi}{\sqrt{1-\epsilon^{2}}} K(\phi), \\
& -12 \eta(U-V)(1-\epsilon \cos \phi)-6 \epsilon(\eta+1) \sin \phi, \\
& \left.+\frac{24 \epsilon(\eta+1)(1-\epsilon \cos \phi) \sin \phi}{\left(2+\epsilon^{2}\right)}\right\},
\end{aligned}
$$

$H_{100}(\phi)=-6 \eta(U-V) \frac{\sqrt{1-\epsilon^{2}}}{(1-\epsilon \cos \phi)^{2}}\left(1+4 \epsilon \sqrt{1-\epsilon^{2}} K(\phi) \sin \phi\right)$,

$M_{100}(\phi)=-6 \eta(U-V) \frac{(\eta+1)\left(1-\epsilon^{2}\right)}{\left(2+\epsilon^{2}\right)(1-\epsilon \cos \phi)^{2}}\left(1+4 \epsilon \sqrt{1-\epsilon^{2}} K(\phi) \sin \phi\right)$.

The general solution of (2.34) is given by

$$
\begin{aligned}
\Psi_{10}(x, \phi)= & \sum_{n=0}^{3} A_{10 n}(\phi) x^{n}-\frac{2 \epsilon\left(1-\epsilon^{2}\right) \sin \phi}{(1-\epsilon \cos \phi)^{2}} \sum_{n=0}^{3} B_{10 n}(\phi) \frac{n !}{(n+4) !} x^{n+4} \\
& +\frac{x^{4}}{7 !}\left(210 V+6(U-V)(7-2 x) x^{2}\right) C_{100}(\phi) \\
& +\frac{2 x^{5}}{7 !}\left(21-7(\eta+2) x+3(\eta+1) x^{2}\right) D_{100}(\phi) \\
& -\frac{2 x^{5}}{6 !}(x-3) E_{100}(\phi)-\frac{2 x^{6}}{7 !}(3 x-7) F_{100}(\phi) \\
& +\frac{2 x^{4}}{7 !}\left(18(\eta+1) x^{3}-7(7 \eta+11) x^{2}+42(\eta+3) x-105\right) H_{100}(\phi) \\
& -\frac{x^{5}}{140}\left(2 x^{2}-7 x+7\right) M_{100}(\phi) .
\end{aligned}
$$


The functions involving $\phi$ are determined so that the boundary conditions (2.35) are satisfied and so that $P_{10}$ is periodic and singlevalued. Thus we have

$$
A_{101}(\phi)=0
$$

$A_{102}(\phi)$

$$
\begin{aligned}
= & -\frac{2 \epsilon\left(1-\epsilon^{2}\right) \sin \phi}{(1-\epsilon \cos \phi)^{2}} \sum_{n=0}^{3} \frac{(n+1) !}{(n+4) !} B_{10 n}(\phi)+\frac{13 U+22 V}{840} C_{100}(\phi)+\frac{24-18 \eta}{7} D_{100}(\phi) \\
& +\frac{1}{120} E_{100}(\phi)+\frac{1}{280} F_{100}(\phi)+\frac{1}{168}(5 \eta-16) H_{100}(\phi)+\frac{33}{280} M_{100}(\phi) \\
& +\frac{3\left(1-\epsilon^{2}\right) \eta}{35\left(2+\epsilon^{2}\right)}\left[\frac{(\eta+1)(13 U-197 V)}{2+\epsilon^{2}}-\frac{118}{3} \eta(U-V)-\frac{2}{3}(31 V+74 U)\right]
\end{aligned}
$$

$A_{103}(\phi)$

$$
\begin{aligned}
= & \frac{2 \epsilon\left(1-\epsilon^{2}\right) \sin \phi}{(1-\epsilon \cos \phi)^{2}} \sum_{n=0}^{3} \frac{(n+2) n !}{(n+4) !} B_{10 n}(\phi)-\frac{(9 U+26 V)}{420} C_{100}(\phi)-\frac{(22-13 \eta)}{2520} D_{100}(\phi) \\
& -\frac{1}{72} E_{100}(\phi)-\frac{26}{7 !} F_{100}(\phi)-\frac{1}{1260}(32 \eta-101) H_{100}(\phi)-\frac{13}{210} M_{100}(\phi) \\
& -\frac{2\left(1-\epsilon^{2}\right) \eta}{35\left(2+\epsilon^{2}\right)}\left[\frac{(\eta+1)(13 U-197 V)}{2+\epsilon^{2}}-\frac{118}{3} \eta(U-V)-\frac{2}{3}(31 V+74 U)\right],
\end{aligned}
$$

$P_{10}(\phi ; \epsilon)$

$$
\begin{aligned}
= & -\frac{3 \epsilon(\eta+1)^{2}}{35\left(2+\epsilon^{2}\right)} \cos \phi+\frac{1}{7}\left(1-\frac{4}{5} \eta+\eta^{2}\right) \log (1-\epsilon \cos \phi)-\frac{27}{35} \frac{(\eta+1)^{2}}{\left(2+\epsilon^{2}\right)^{2}}(1-\epsilon \cos \phi)^{2} \\
& +\frac{26 V+9 U}{70} S_{1}(\phi)-\frac{13 \eta-22}{420} S_{2}(\phi)+\frac{1}{12} S_{3}(\phi) \frac{13}{420} S_{4}(\phi)+\frac{32 \eta-101}{210} S_{5}(\phi) \\
& +\frac{13}{35} S_{6}(\phi)+\frac{12}{\sqrt{1-\epsilon^{2}}} \eta^{2} V(U-V) S_{7}(\phi)-\frac{6 \eta(\eta+1)}{2+\epsilon^{2}} V S_{8}(\phi)+2 \eta(\eta+2) V \phi \\
& +\left(\frac{12 \eta(\eta+1)(13 U-197 V)}{35\left(2+\epsilon^{2}\right)^{2}}-\frac{1472 \eta^{2}(U-V)}{35\left(2+\epsilon^{2}\right)}-\frac{24 \eta(31 V+74 U)}{105\left(2+\epsilon^{2}\right)}\right) \\
& \times\left(\frac{2+\epsilon^{2}}{2} \phi-2 \epsilon \sin \phi+\frac{\epsilon^{2}}{4} \sin (2 \phi)\right),
\end{aligned}
$$

where

$$
\begin{aligned}
S_{1}(\phi)= & -\frac{24 \eta^{2}}{\sqrt{1-\epsilon^{2}}}(U-V)\left[L(\phi)-\epsilon K(\phi) \sin \phi+\frac{1}{2} \sqrt{1-\epsilon^{2}} \log (1-\epsilon \cos \phi)\right] \\
& +6 \eta(\eta+1) \phi+\frac{12 \eta(\eta+1)}{2+\epsilon^{2}}(\phi-\epsilon \sin \phi), \\
S_{2}(\phi)= & -\frac{24 \epsilon \eta}{\sqrt{1-\epsilon^{2}}}(U-V)\left[-\cos \phi K(\phi)-\sqrt{1-\epsilon^{2}} \phi+\frac{1}{\epsilon} K(\phi)\right],
\end{aligned}
$$




$$
\begin{aligned}
S_{3}(\phi)= & -\frac{144 \eta^{2}}{1-\epsilon^{2}}(U-V)^{2}\left[(1-\epsilon \cos \phi)^{2} K(\phi)^{2}-\frac{\sqrt{1-\epsilon^{2}}}{2} L(\phi)+\frac{\epsilon}{2} K(\phi) \sin \phi\right. \\
& \left.-\frac{1}{4}\left(1-\epsilon^{2}\right) \log (1-\epsilon \cos \phi)\right] \\
& -\frac{24 \eta(\eta+2)}{\sqrt{1-\epsilon^{2}}}(U-V)\left[-\epsilon K(\phi) \cos \phi-\frac{\sqrt{1-\epsilon^{2}}}{4} \phi+\frac{\epsilon \sqrt{1-\epsilon^{2}}}{4} \sin \phi\right] \\
S_{4}(\phi)= & -\frac{576 \eta^{2}}{2\left(1-\epsilon^{2}\right)}(U-V)^{2}\left(K(\phi)^{2}\right)(1-\epsilon \cos \phi)^{2}-\frac{144 \eta^{2}(U-V)}{\sqrt{1-\epsilon^{2}}} \\
& \times\left[L(\phi)-\epsilon K(\phi) \sin \phi+\frac{1-\epsilon^{2}}{2} \log (1-\epsilon \cos \phi)\right] \\
& +\frac{72 \eta(\eta+1)}{\sqrt{1-\epsilon^{2}}(U-V)\left[-\frac{\sqrt{1-\epsilon^{2}}}{2} \phi+K(\phi)(1-\epsilon \cos \phi)\right]} \\
& -\frac{288 \eta(\eta+1)}{\left(2+\epsilon^{2}\right) \sqrt{1-\epsilon^{2}}}(U-V)\left[-\frac{\sqrt{1-\epsilon^{2}}}{4}+\frac{1}{2} K(\phi)(1-\epsilon \cos \phi)^{2}+\frac{\epsilon}{4} \sin \phi\right], \\
S_{5}(\phi)= & -24 \eta(U-V)\left[(1-\epsilon \cos \phi) K(\phi)-\frac{\sqrt{1-\epsilon^{2}}}{2} \phi\right]-\frac{6 \eta(U-V)}{\sqrt{1-\epsilon^{2}}} \phi \\
S_{6}(\phi)= & -\frac{24 \eta(\eta+1)(U-V) \sqrt{1-\epsilon^{2}}}{\left(2+\epsilon^{2}\right)}\left[(1-\epsilon \cos \phi) K(\phi)-\frac{\phi}{2}\right] \\
& -\frac{6 \eta(\eta+1)}{2+\epsilon^{2}}(U-V) \phi, \\
S_{7}(\phi)= & L(\phi)-\epsilon K(\phi) \sin \phi+\frac{\sqrt{1-\epsilon^{2}}}{2} \log (1-\epsilon \cos \phi) \\
S_{8}(\phi)= & \phi-\epsilon \sin \phi .
\end{aligned}
$$

We observe that the stream function $\Psi_{10}$ is antisymmetric and the pressure is symmetric about the line $\phi=0$.

THE CURVATURE OR $\alpha$ CORRECTION: $\alpha \neq 0, R_{M}=0$. The right-hand side of (2.37) is given by

$$
\frac{48 \eta(U-V) K(\phi)}{(1-\epsilon \cos \phi)}\left(3-3 \epsilon \cos \phi-2 \sqrt{1-\epsilon^{2}}\right)+24 B_{010}(\phi, \epsilon)
$$

where

$$
\begin{aligned}
B_{010}(\phi, \epsilon) & =\frac{\epsilon \sqrt{1-\epsilon^{2}}(\eta+1)(2 \cos \phi+\epsilon)\left(3-3 \epsilon \cos \phi-2 \sqrt{1-\epsilon^{2}}\right)}{2\left(2+\epsilon^{2}\right)(1-\epsilon \cos \phi)^{2}}, \\
\Psi_{01}(x, \phi) & =\sum_{n=0}^{3} A_{01 n}(\phi) x^{n}+\left[B_{010}(\phi)-(U-V) C_{010}(\phi)\right] x^{4}, \\
C_{010}(\phi) & =-\frac{2 \eta K(\phi)}{(1-\epsilon \cos \phi)}\left(3-3 \epsilon \cos \phi-2 \sqrt{1-\epsilon^{2}}\right),
\end{aligned}
$$




$$
\begin{aligned}
A_{010}(\phi)= & S_{010}+a_{010}, \\
A_{011}(\phi)= & M(\phi)=-\frac{\epsilon(\epsilon-\cos \phi)}{2(1-\epsilon \cos \phi)^{2}}, \\
A_{012}(\phi)= & -2 M(\phi)-N(\phi)+B_{010}(\phi)-(U-V) C_{010}(\phi)-3 S_{010} \\
& +3\left[\frac{\eta U \epsilon \sin \phi}{2(1-\epsilon \cos \phi)}+\frac{2 \eta U K(\phi)}{\sqrt{1-\epsilon^{2}}}+\frac{1-\epsilon^{2}}{3\left(2+\epsilon^{2}\right)}-\frac{(\eta+1)\left(4-\epsilon^{2}-3 \sqrt{1-\epsilon^{2}}\right)}{6\left(2+\epsilon^{2}\right)}\right],
\end{aligned}
$$

$$
\begin{aligned}
A_{013}(\phi)= & M(\phi)+N(\phi)+2 B_{010}(\phi)-2(U-V) C_{010}(\phi)+2 S_{010} \\
& -2\left[\frac{\eta U \epsilon \sin \phi}{2(1-\epsilon \cos \phi)}+\frac{2 \eta U K(\phi)}{\sqrt{1-\epsilon^{2}}}+\frac{1-\epsilon^{2}}{3\left(2+\epsilon^{2}\right)}-\frac{(\eta+1)\left(4-\epsilon^{2}-3 \sqrt{1-\epsilon^{2}}\right)}{6\left(2+\epsilon^{2}\right)}\right],
\end{aligned}
$$

$$
S_{010}(\phi)=-\frac{\eta V \epsilon \sin \phi}{2(1-\epsilon \cos \phi)}
$$

and the pressure is as follows:

$$
\begin{aligned}
P_{01}(\phi ; \epsilon)= & {\left[-\frac{8 \epsilon}{2+\epsilon^{2}}+(\eta+1)\left(-\frac{\epsilon}{1-\epsilon^{2}}+\frac{6 \epsilon\left(2-\epsilon^{2}\right)}{\left(2+\epsilon^{2}\right) \sqrt{1-\epsilon^{2}}}\right)\right] \sin \phi } \\
& +\left[\frac{\epsilon^{2}}{2+\epsilon^{2}}+\frac{\eta+1}{2}\left(\frac{\epsilon^{2}}{1-\epsilon^{2}}-\frac{6 \epsilon^{2}}{\left(2+\epsilon^{2}\right) \sqrt{1-\epsilon^{2}}}\right) \sin 2 \phi\right] \\
& +\left[\frac{6 \eta(U-V)}{\left(1-\epsilon^{2}\right)^{3 / 2}}\left(2 \epsilon^{2}+6 \sqrt{1-\epsilon^{2}}+3 \epsilon^{2} \sqrt{1-\epsilon^{2}}-4\right)+\frac{12 \eta U\left(2+\epsilon^{2}\right)}{\left(1-\epsilon^{2}\right)^{3 / 2}}\right] L(\phi) \\
& +\frac{24 \epsilon \eta}{\left(1-\epsilon^{2}\right)^{3 / 2}} \sin \phi K(\phi)\left(2-3 \sqrt{1-\epsilon^{2}}(U-V)-2 U\right) \\
& +\frac{3 \eta}{\left(1-\epsilon^{2}\right)} \log (1-\epsilon \cos \phi)\left(-3\left(2-3 \sqrt{1-\epsilon^{2}}(U-V)+2(3 U+V)\right)\right) \\
& +\frac{3 \eta \epsilon^{2}}{\left(1-\epsilon^{2}\right)^{3 / 2}} \sin 2 \phi K(\phi)\left(-\left(2-3 \sqrt{1-\epsilon^{2}}(U-V)+2 U\right)\right) \\
& +\frac{3 \eta \epsilon}{\left(1-\epsilon^{2}\right)} \cos \phi\left(2-3 \sqrt{1-\epsilon^{2}}(U-V)-4 U\right) \\
& +\frac{3 \eta U \epsilon^{2}}{2\left(1-\epsilon^{2}\right)} \cos 2 \phi+\frac{6 \eta V}{\left(1-\epsilon^{2}\right)}+\frac{9 \eta(U-V)}{\sqrt{1-\epsilon^{2}}} .
\end{aligned}
$$

As in the Reynolds approximation, $\Psi_{01}$ is symmetric and the pressure $P_{01}$ is antisymmetric about the line $\phi=0$.

4. Results and discussions. Having calculated the streamfunction and pressure distribution, graphs are plotted to study the effect of suction/injection on the flow values of eccentricity ranging between 0.3 and 0.5 with $\eta\left(=q_{2} / q_{1}\right)$ the ratio of the 
velocity of the outer to the inner cylinder to be 2 and -1 . Figures $1,2,3,4$, and 5 display the contour plot of the stream function $\Psi(x, \phi)$ for values of $\epsilon=0.3, \eta=2$ and $R_{M}=0.01$. It has been observed that the streamlines are almost circular following the contour of the body. Graphs clearly reveal that change in the value of $U$ and $V$ does not affect the flow pattern for a small value of $\epsilon$ with $\eta=2.0$. Figures $6,7,8,9$, and 10 show the streamline pattern for the flow between counter rotating cylinders. Separation is observed in all cases obtained by varying the signs of $U$ and $V$. It is seen that the outer cylinder drags more fluid with itself than the inner cylinder. As the values of $U$ and $V$ vary the flow pattern gets slightly deviated with a change in the sense of movement. As the values of $U$ and $V$ vary the flow pattern gets slightly deviated with a change in the sense of movement. Now from Figures 1, 2, 3, 4, 5, 6, 7, 8,9 , and 10 it can be concluded that when the cylinders rotate with equal speeds but

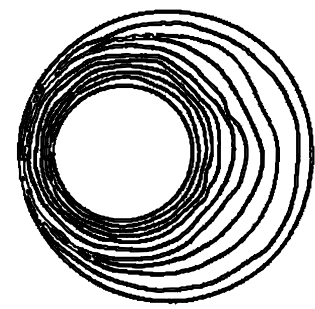

(1) $U=V=0$.

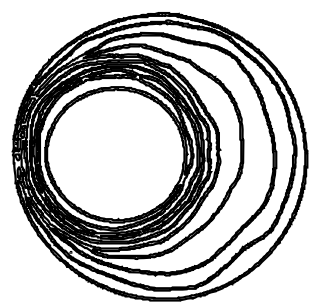

(3) $U=-0.133682 ; V=0.2661364$.

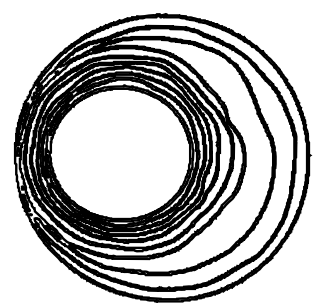

(2) $U=0.133682 ; V=0.2661364$.

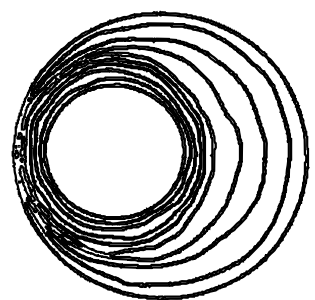

(4) $U=0.133682 ; V=-0.2661364$.

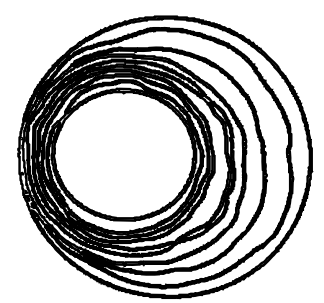

(5) $U=-0.133682 ; V=-0.2661364$.

Figures $1,2,3,4$, and 5 give the streamlines for $\epsilon=0.3 ; \eta=2.0 ; R_{M}=$ $0.01 ; \alpha=0.939368$. 
in opposite direction, the effect of suction/injection is felt even for a small value of the eccentricity parameter.

Now increasing the eccentricity parameter $\epsilon$ to 0.5 , as in the previous case, the streamlines are drawn for the same values of $\eta$, that is, 2 and -1 . For $\eta=2$ the streamline pattern is shown in Figures 11, 12, 13, 14, and 15. The streamline patterns show a small deviation from the usual circular pattern. Comparing the same with the case for which $\epsilon=0.3$, it has been observed that eccentricity plays a vital role in flow separation. The pattern remains unaltered for various values of $U$ and $V$ except with a change in the sense of movement. Finally, from Figures 16, 17, 18, 19, and 20 we observe a clear separation which encircles the inner cylinder. There is a slight variation

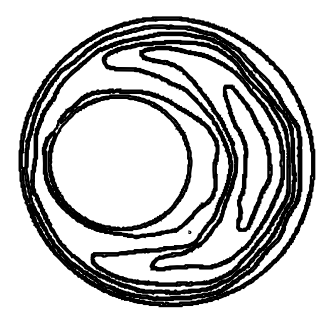

(6) $U=V=0$.

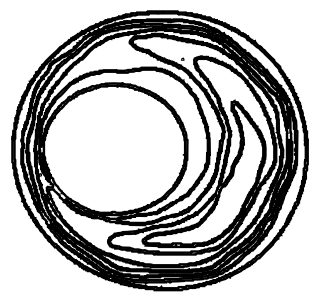

(8) $U=-0.2661364 ; V=0.5322728$.

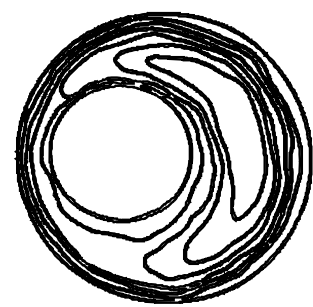

(7) $U=0.2661364 ; V=0.5322728$.

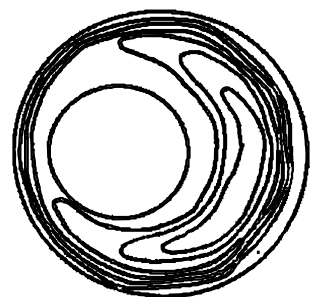

(9) $U=0.2661364 ; V=-0.5322728$.

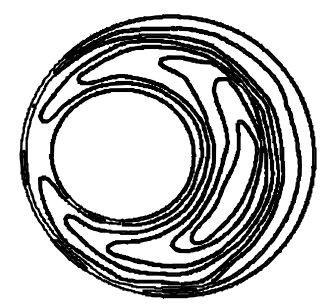

(10) $U=-0.2661364 ; V=-0.5322728$.

Figures $6,7,8,9$, and 10 give the streamlines for $\epsilon=0.3 ; \eta=-1.0 ; R_{M}=0.01 ; \alpha=$ 0.939368 . 
in the separated flow region which gets shifted in accordance to the values assigned to $U$ and $V$.

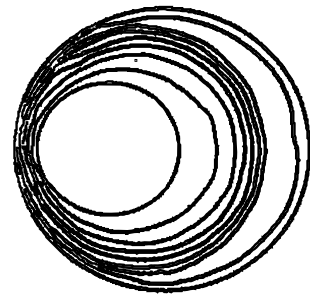

(11) $U=V=0$.

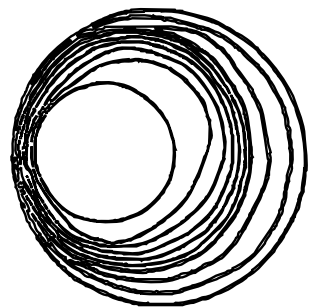

(13) $U=-0.1509782 ; V=0.3019564$.

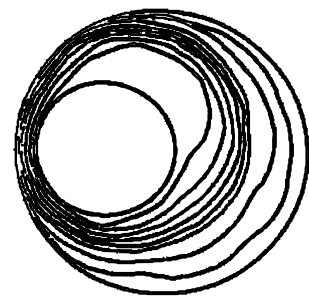

(12) $U=0.1509782 ; V=0.3019564$.

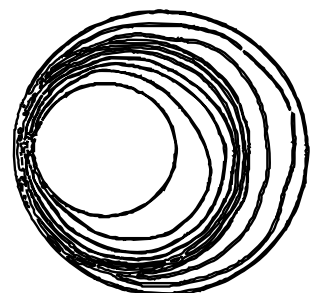

(14) $U=0.1509782 ; V=-0.3019564$.

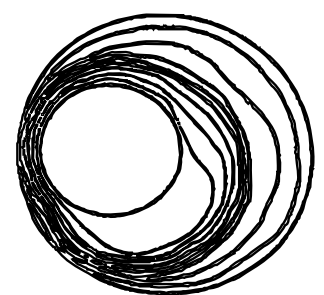

(15) $U=-0.1509782 ; V=-0.3019564$.

Figures $11,12,13,14$, and 15 give the streamlines for $\epsilon=0.5 ; \eta=2.0 ; R_{M}=$ $0.01 ; \alpha=0.827934$.

Figures 21(a-e), 22(a-e), 23(a-e), and 24(a-e) show the corresponding pressure curves for various physical parameters. For $\epsilon=0.3, \eta=2, R_{M}=0.01$ and with $U=$ $V=0$, that is, the case where there is no suction/injection. Figure 21 a reveals that as $\theta$ increases, pressure increases to a certain limit $(\theta=180)$ and then gradually decreases indicating the fact that pressure is periodic in nature. Figures $21 \mathrm{~b}$ and $21 \mathrm{c}$ show the pressure plots for the $U<V$. There is a fall in the magnitude pressure as in comparison with Figure 21a. For $U>V$, there is a slight rise in the magnitude of 
pressure as observed in Figures 21d and 21e. Figures 21a, 21b, 21c, 21d, and 21e are clubbed into a single graph (Figure 21(a-e)) indicating the effect of suction/injection for $\epsilon=0.3$ and $\eta=2.0$. This graph clearly reveals the periodic nature of pressure.

As discussed in the above case, the pressure graphs are drawn for $\epsilon=0.3, \eta=-1$, $R_{M}=0.01$. Here the cylinders rotate with equal speed in opposite directions. Thus eventhough the patterns remain alike here the trend is reversed. This is seen from Figures 22a, 22b, 22c, 22d, and 22e. Similarly changing the values of the eccentricity parameter to 0.5 , the pressure distribution is shown for $\eta=2$ and -1 in Figures 21(ae), 22(a-e), 23(a-e), and 24(a-e).

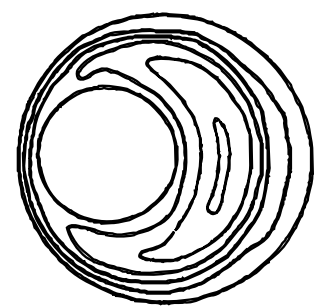

(16) $U=V=0$.

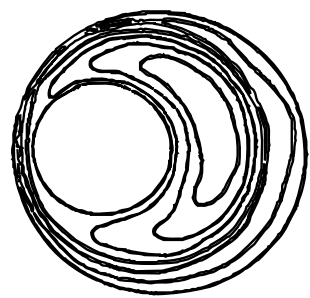

(18) $U=-0.3019564 ; V=0.6039128$.

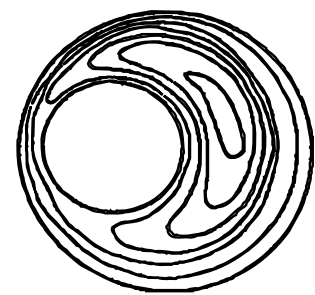

(17) $U=0.3019564 ; V=0.6039128$.

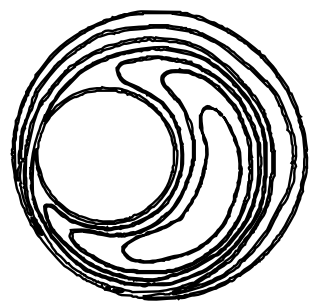

(19) $U=0.3019564 ; V=-0.6039128$.

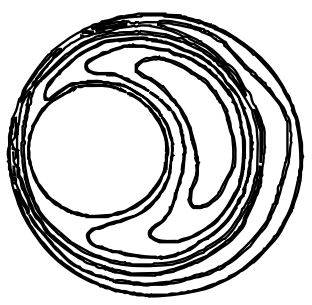

(20) $U=-0.3019564 ; V=-0.6039128$.

Figures $16,17,18,19$, and 20 give the streamlines for $\epsilon=0.5 ; \eta=-1.0 ; R_{M}=$ $0.01 ; \alpha=0.939368$. 


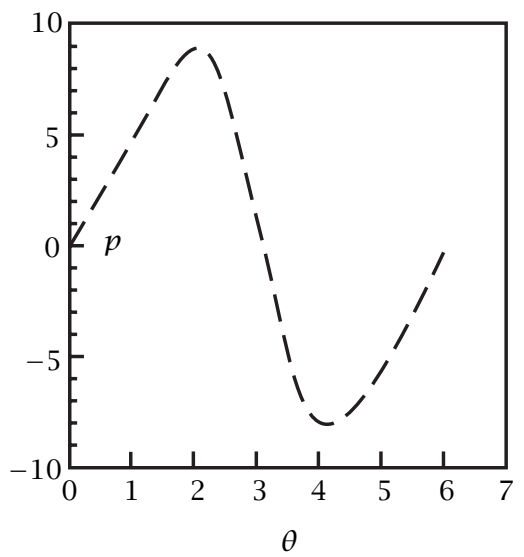

(a) $U=V=0$.

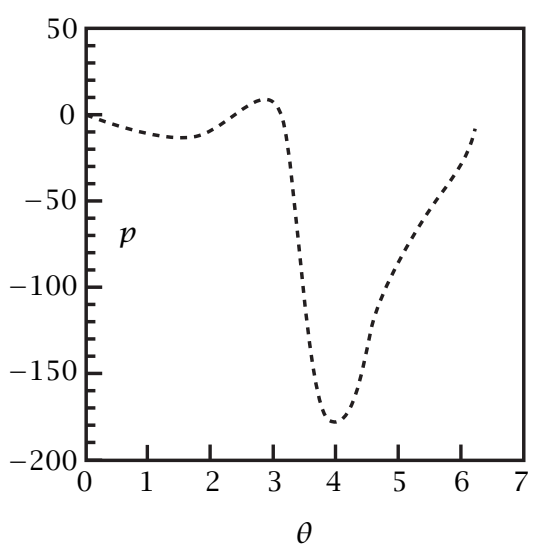

(c) $U=-0.133682 ; V=0.2661364$.

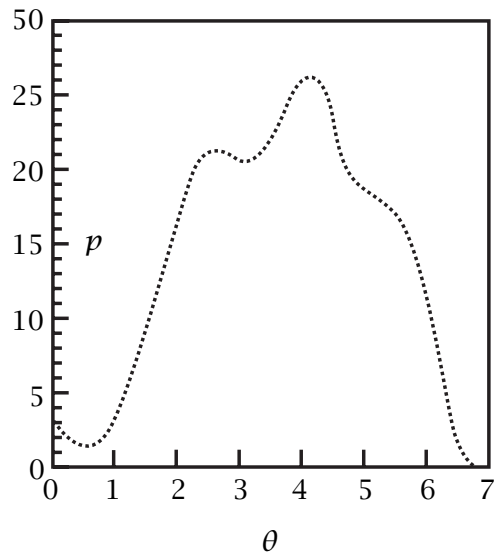

(e) $U=-0.133682 ; V=-0.2661364$.

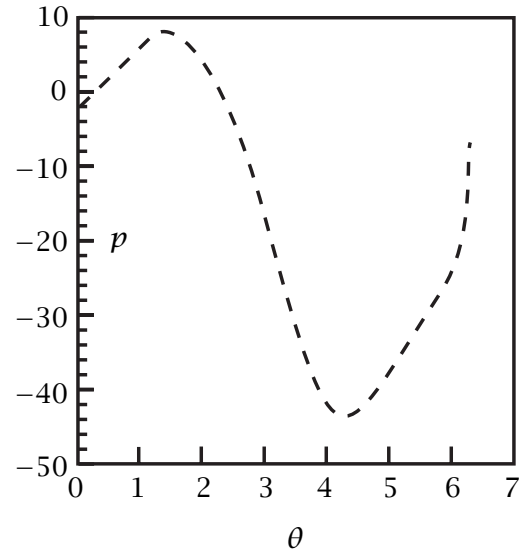

(b) $U=0.133682 ; V=0.2661364$.

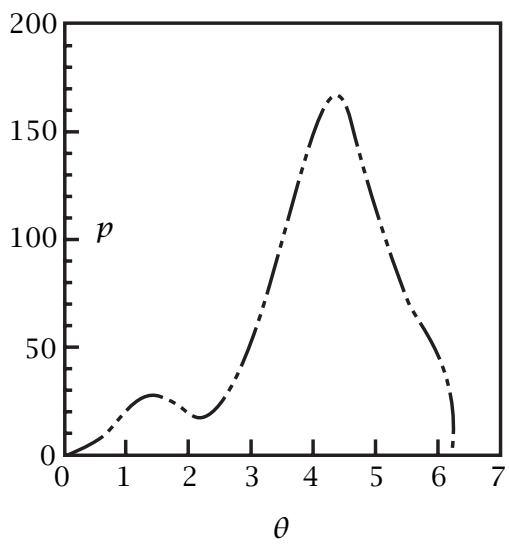

(d) $U=0.133682 ; V=-0.2661364$.

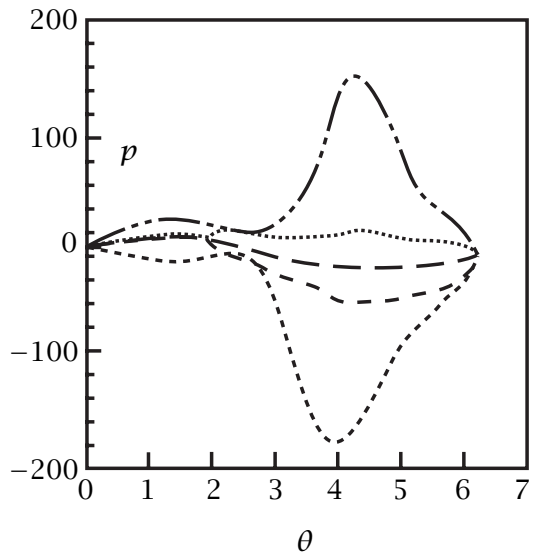

(a-e)

FIGURE 21. The pressure distribution for $\epsilon=0.3 ; \eta=2.0 ; R_{M}=0.01 ; \alpha=0.939368$. 


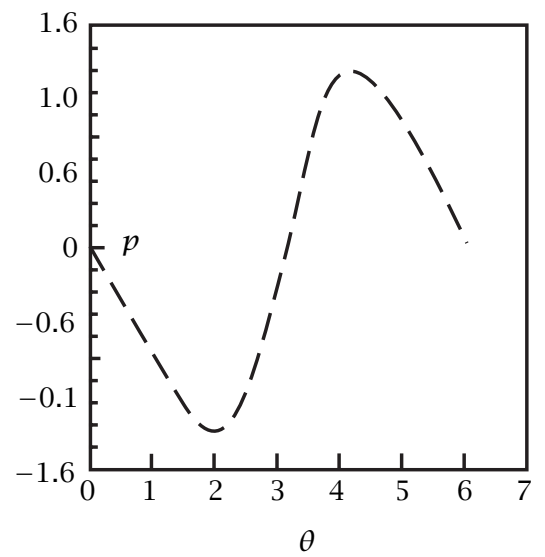

(a) $U=V=0$.

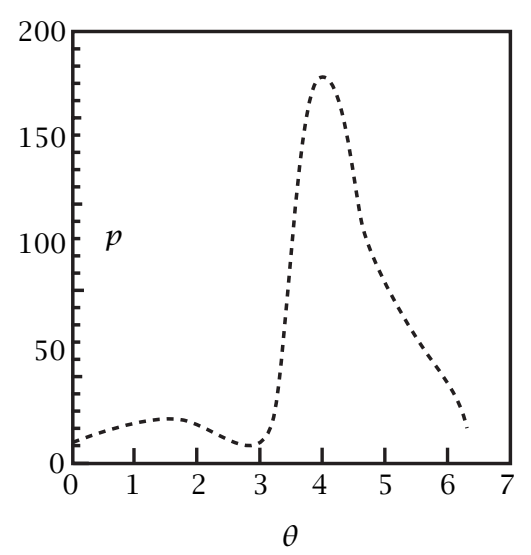

(c) $U=-0.2661364 ; V=0.5322728$.

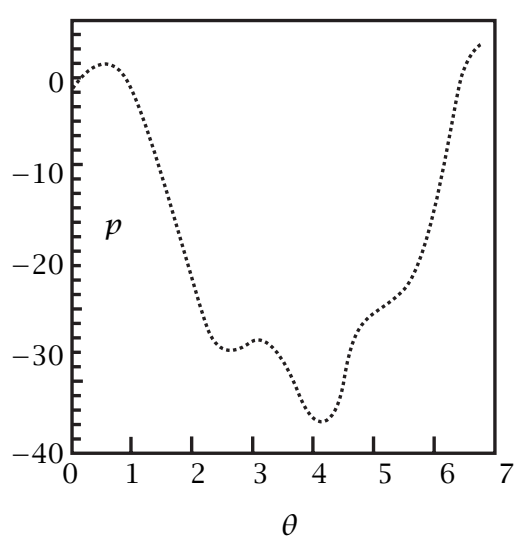

(e) $U=-0.2661364 ; V=-0.5322728$.

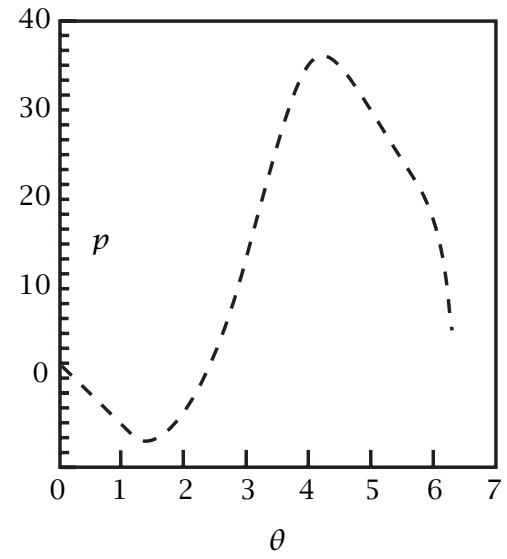

(b) $U=0.2661364 ; V=0.5322728$.

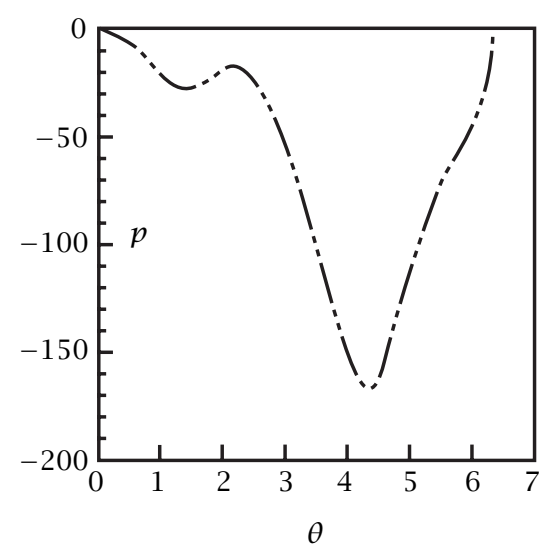

(d) $U=0.2661364 ; V=-0.5322728$.

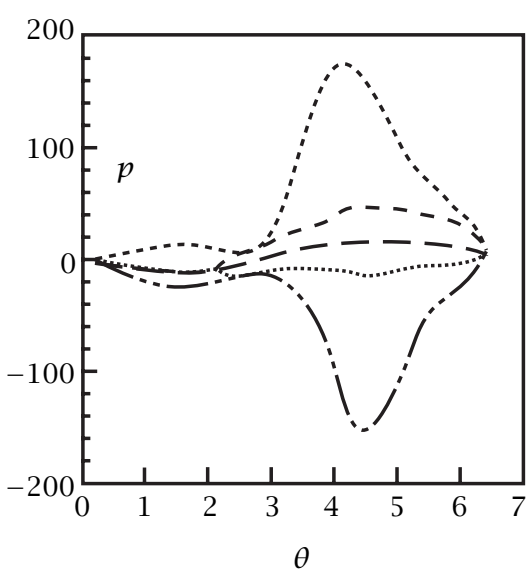

(a-e)

FIGURE 22. The pressure distribution for $\epsilon=0.3 ; \eta=-1.0 ; R_{M}=0.01 ; \alpha=0.939368$. 


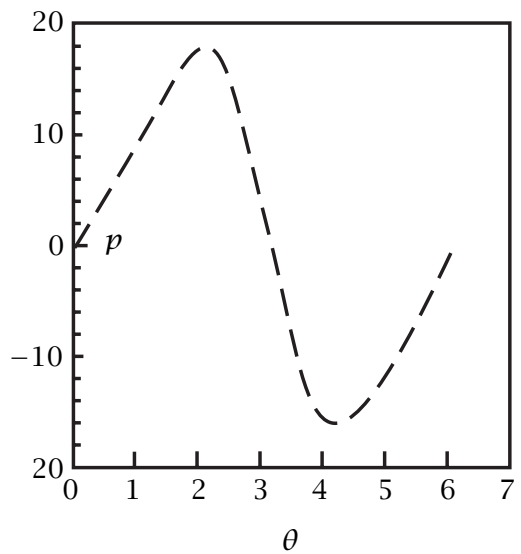

(a) $U=V=0$.

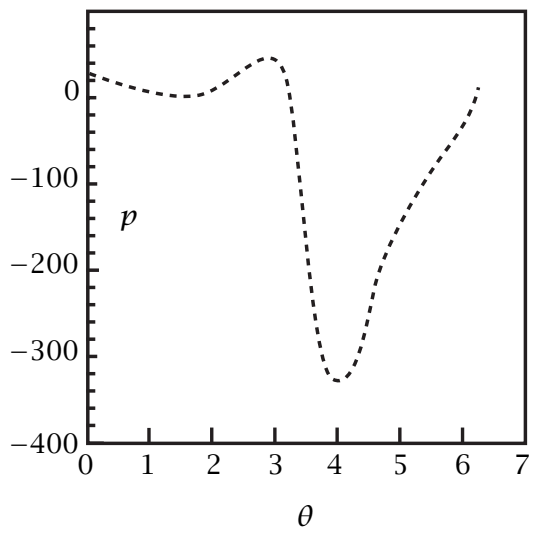

(c) $U=-0.1509782 ; V=0.3019564$.

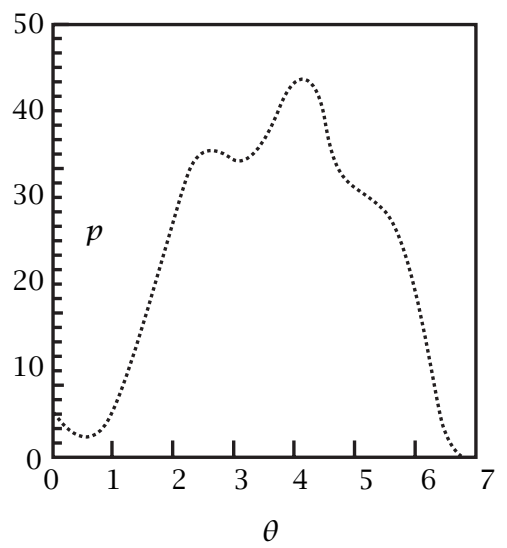

(e) $U=-0.1509782 ; V=-0.3019564$.

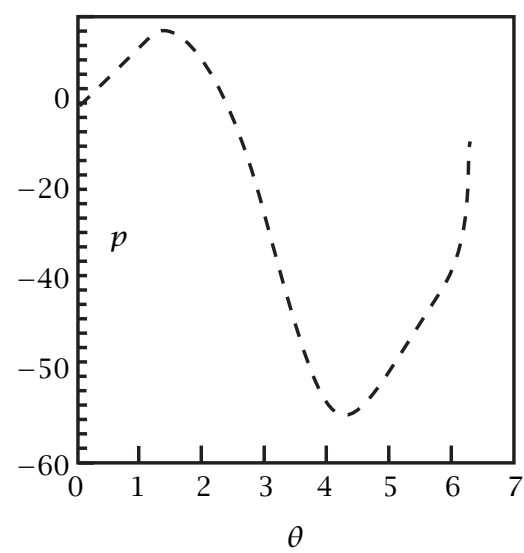

(b) $U=0.1509782 ; V=0.3019564$.

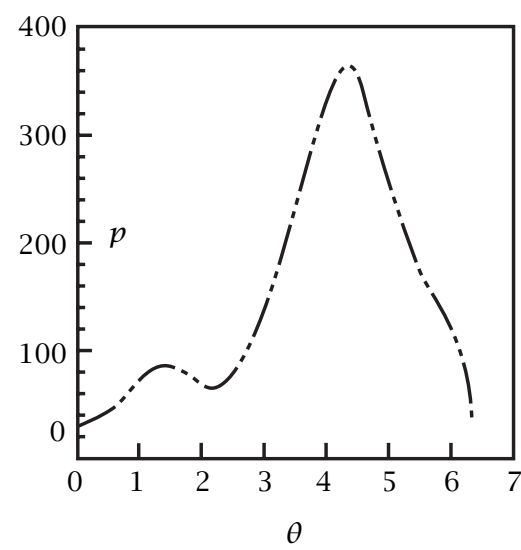

(d) $U=0.1509782 ; V=-0.3019564$.

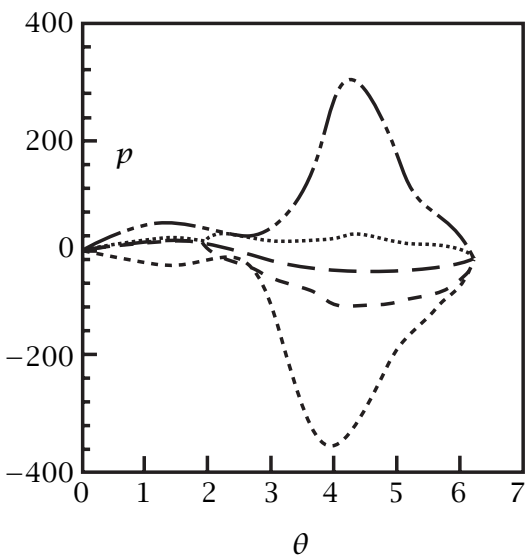

(a-e)

FIGURE 23. The pressure distribution for $\epsilon=0.5 ; \eta=2.0 ; R_{M}=0.01 ; \alpha=0.827934$. 


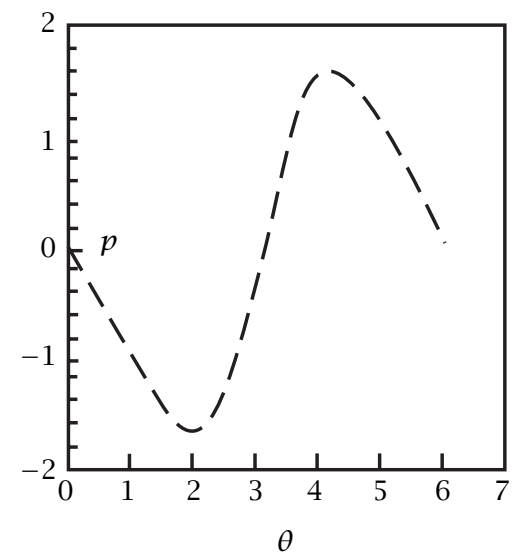

(a) $U=V=0$.

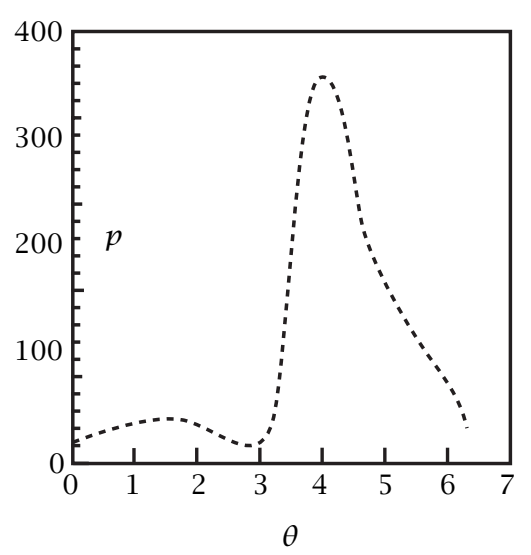

(c) $U=-0.3019564 ; V=0.6039128$.

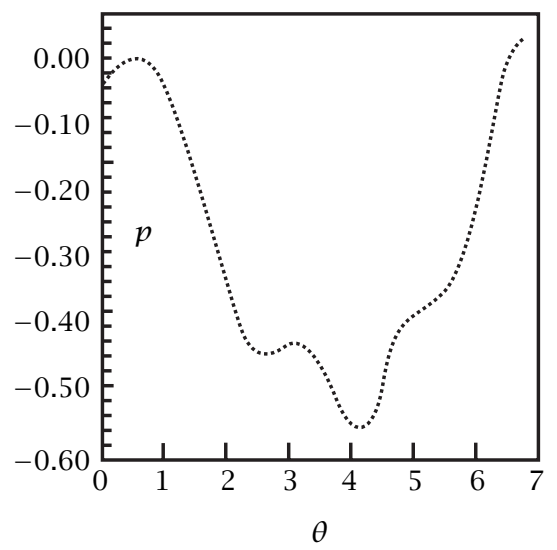

(e) $U=-0.3019564 ; V=-0.6039128$.

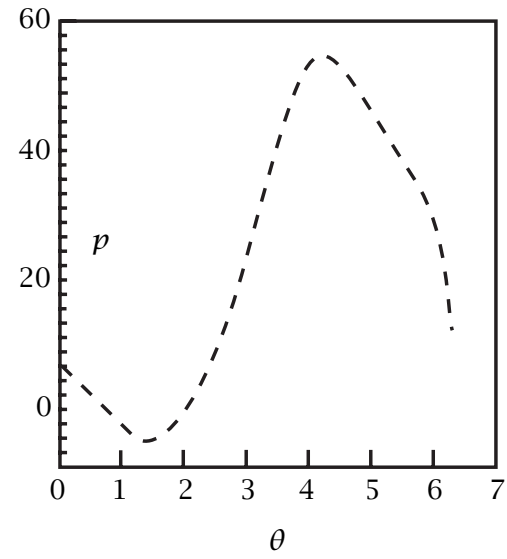

(b) $U=0.3019564 ; V=0.6039128$.

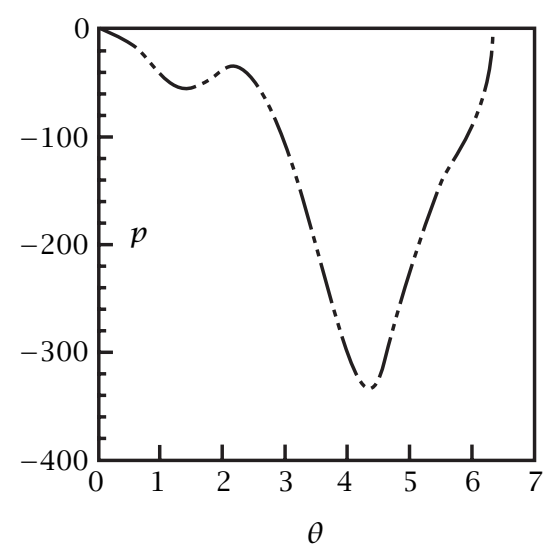

(d) $U=0.3019564 ; V=-0.6039128$.

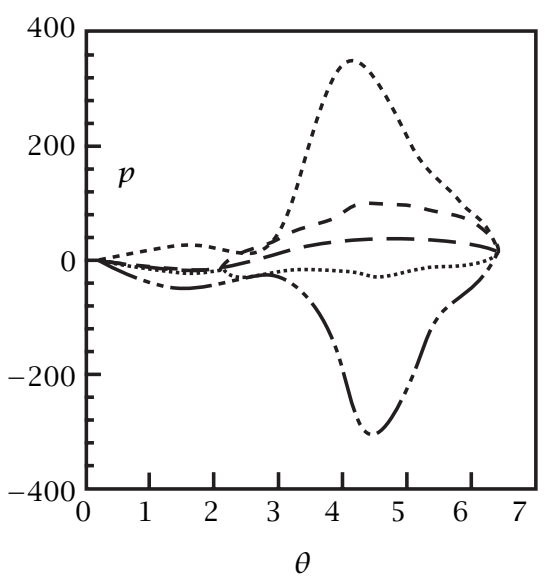

(a-e)

FIGURE 24. The pressure distribution for $\epsilon=0.5 ; \eta=-1.0 ; R_{M}=0.01 ; \alpha=0.939368$. 
ACKNOWLEDGement. S. Meena thanks CSIR, New Delhi for the financial assistance through a Senior Research Fellowship during the present work.

\section{REFERENCES}

[1] P. Chandra, Effect of cartilage porosity on synovial joint lubrication, Proc. N. A. Sc. (1979), 265-279.

[2] L. Dintenfass, Lubrication in synovial joints, Archeological approach to the problem of joint movement and joint lubrication, J. Bone Int. Surg. 45A (1963), 1241-1253.

[3] R. C. DiPrima and J. T. Stuart, Flow between eccentric rotating cylinders, J. Lub. Tech. Trans. ASME Series F 72 (1972), 266-274.

[4] D. Dowson, Modes of lubrication in human joints, Proc. Inst. Mech. Engrs. 181 (1967), 45-53, 3J.

[5] R. S. Fein, Are synovial joints squeeze-film lubricated?, Proc. Inst. Mech. Engrs. 181 (1967), 125-132, 3J.

[6] M. M. Kamal, Separation in the flow between eccentric rotating cylinders, ASME Journal of Basic Engineering 88 (1966), 717-724.

[7] E. S. Kulinski and S. Ostrach, Journal-bearing velocity profiles for small-eccentricity and moderated modified Reynolds numbers, J. Appl. Mech. 34 (1967), 15-22. Zbl 158.23503.

[8] F. F. Ling, A new model of articular cartilage in human joints, J. Lub. Tech. 96F (1974), no. 3, 449-454.

[9] C. W. McCutchen, More on weeping lubrication, Inst. Mech. Engrs. 121 (1969), 117-123.

[10] O. Reynolds, On the theory of lubrication and its application to Mr. Beuchamp Tower's experiments, Phil. Trans. Roy. Soc. 177 (1886), 157-234.

[11] P. Sambasiva Rao, B. Srivathsa, and P. N. Murthy, A numerical scheme for hydrodynamic lubrication of synovial fluid, Indian J. pure appl. Math. 26 (1995), 831-835.

[12] D. R. Sood and H. G. Elrod, On the flow between two long eccentric cylinders, Tech. Report 17, Lubrication Research Laboratory, Columbia University, 1970.

[13] G. H. Wannier, A contribution to the hydrodynamics of lubrication, Quart. Appl. Math. 8 (1950), 1-32. MR 12,217m. Zbl 036.25804.

[14] W. W. Wood, The asymptotic expansions at large Reynolds numbers for steady motion between noncoaxial rotating cylinders, J. Fluid Mech. 3 (1957), 159-175. MR 19,1119f. Zbl 081.19304.

S. MEena AND P. Kandaswamy: Department of Mathematics, Bharathiar UniVersity, COIMBATORE-641 046, INDIA

Lokenath Debnath: Department of Mathematics, University of Central Florida, ORLANDO, FLORIDA 32816, USA

E-mail address: 1debnath@pegasus.cc.ucf.edu 


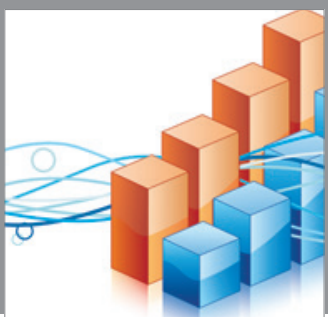

Advances in

Operations Research

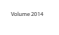

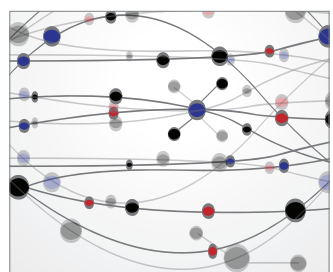

\section{The Scientific} World Journal
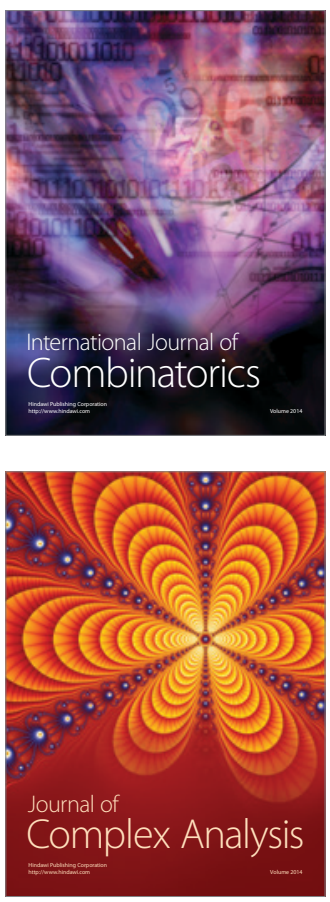

International Journal of

Mathematics and

Mathematical

Sciences
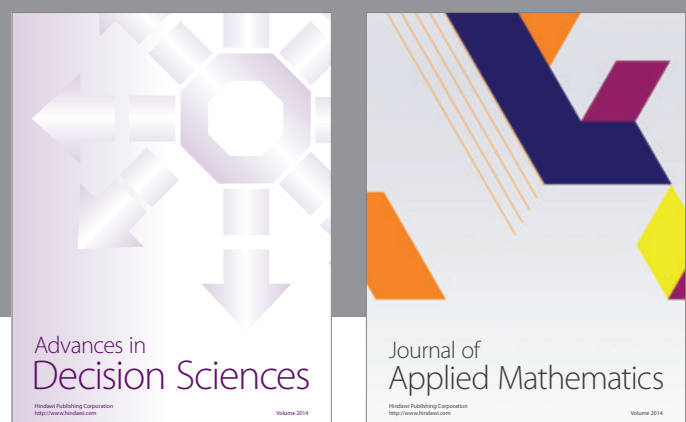

Journal of

Applied Mathematics
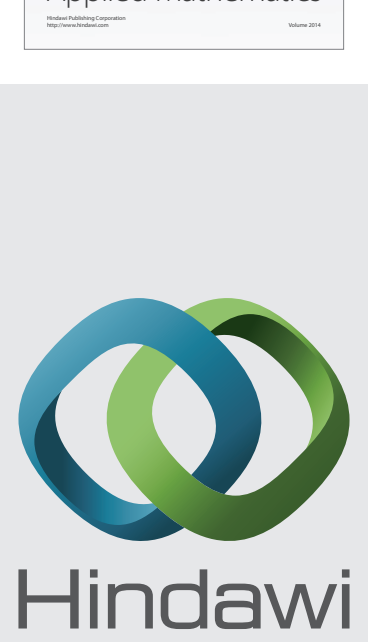

Submit your manuscripts at http://www.hindawi.com
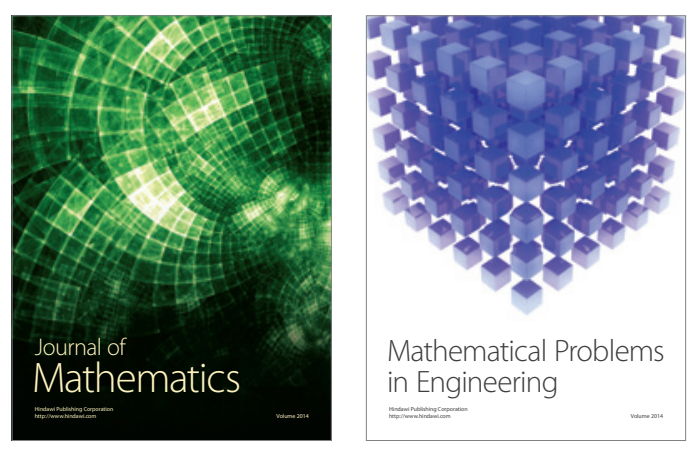

Mathematical Problems in Engineering
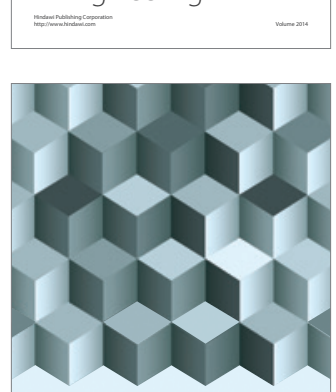

Journal of

Function Spaces
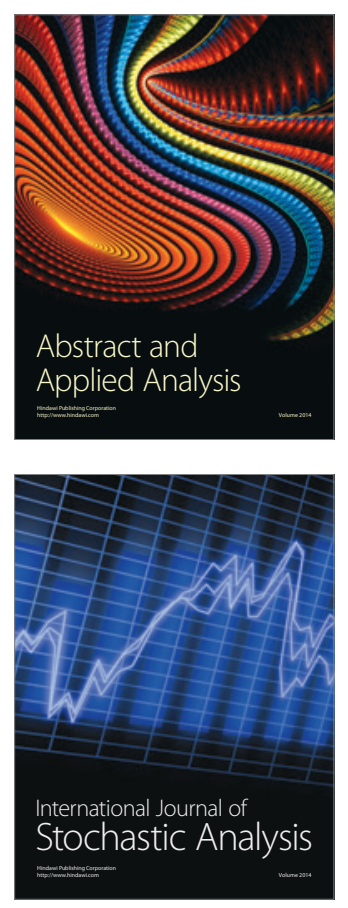

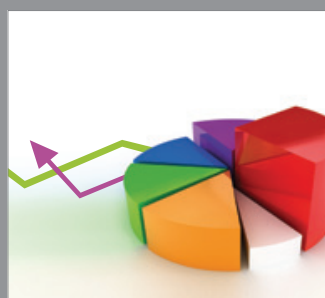

ournal of

Probability and Statistics

Promensencen
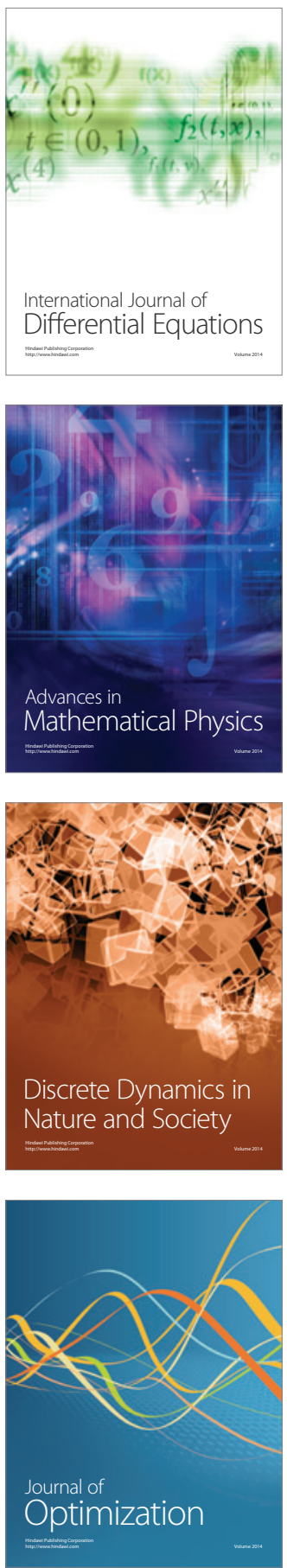